\title{
Algebraic Simplification Techniques for Propositional Satisfiability
}

\author{
João Marques-Silva \\ Department of Informatics, Technical University of Lisbon, \\ IST/INESC/CEL, Lisbon, Portugal \\ jpms@inesc.pt
}

\begin{abstract}
The ability to reduce either the number of variables or clauses in instances of the Satisfiability problem (SAT) impacts the expected computational effort of solving a given instance. This ability can actually be essential for specific and hard classes of instances. The objective of this paper is to propose new simplification techniques for Conjunctive Normal Form (CNF) formulas. Experimental results, obtained on representative problem instances, indicate that large simplifications can be observed.
\end{abstract}

\section{Introduction}

Recent years have seen the proposal of several effective algorithms for solving Propositional Satisfiability (SAT), that include, among others, local search and variations, backtrack search improved with different search pruning techniques, backtrack search with randomization and restarts, continuous formulations and algebraic manipulation. (These different algorithms are further described and cited in [3].) Moreover, these algorithms have allowed efficiently solving different classes of instances of SAT. It is generally accepted that whereas most algorithms for solving SAT can be competitive in proving satisfiability for different classes of instances, backtrack search is preferred when the objective is to prove unsatisfiability. Nevertheless, algebraic simplification solutions are also known to be competitive for proving unsatisfiability in specific contexts [2].

The main goal of this paper is to propose and categorize new simplification techniques, and illustrate the effectiveness of algebraic simplification as a preprocessing tool for SAT algorithms. Moreover, we illustrate the application of the proposed simplification techniques in real-world instances of SAT.

The paper is organized as follows. We start with a few definitions in Section 2. Next we address algebraic simplification, namely the techniques in this paper. Section 4 provides experimental results on applying the proposed simplification techniques on real-world instances of SAT. Finally, Section 5 concludes the paper.

\section{Definitions}

This section introduces the notational framework used throughout the paper. Propositional variables are denoted $x_{1}, \ldots, x_{n}$, and can be assigned truth values 0 
(or $F$ ) or 1 (or $T$ ). In addition to letter $x$, and whenever necessary, we will use letters $y, w$ and $z$ to denote variables. To denote specific variables we may also use $x_{i}, x_{j}, x_{k}, \ldots$ A literal $l$ is either a variable $x_{i}$ (i.e. a positive literal) or its complement $\neg x_{i}$ (i.e. a negative literal). A clause $\omega$ is a disjunction of literals and a CNF formula $\varphi$ is a conjunction of clauses. When referring to specific clauses we will utilize subscripts $a, b, \ldots$, and when referring to sub-formulas of a CNF formula we will utilize subscripts $r, s, \ldots$. Disjunctions of literals, not necessarily representing clauses will be represented as $\alpha, \beta, \gamma, \delta, \epsilon$.

\section{Algebraic Simplification}

Different formula simplification techniques have been proposed over the years. A detailed account of these techniques if provided in [3]. In this section we concentrate on two new techniques, namely support-set variable equivalence and inference of binary clauses.

\subsection{Support Set Variable Equivalence}

In many practical situations, a sub-formula $\varphi_{s}$ of a CNF formula $\varphi$ actually describes a Boolean function $x_{i}=f\left(y_{1}, \ldots, y_{k}\right)$. For example, the sub-formula $\varphi_{a}=\left(y_{1} \vee \neg x\right) \wedge\left(y_{2} \vee \neg x\right) \wedge\left(\neg y_{1} \vee \neg y_{2} \vee x\right)$ describes the Boolean function $x=y_{1} \wedge y_{2}$. More interestingly, if we have two sub-formulas $\varphi_{a}=\left(y_{1} \vee \neg x\right) \wedge$ $\left(y_{2} \vee \neg x\right) \wedge\left(\neg y_{1} \vee \neg y_{2} \vee x\right)$ and $\varphi_{b}=\left(y_{1} \vee \neg z\right) \wedge\left(y_{2} \vee \neg z\right) \wedge\left(\neg y_{1} \vee \neg y_{2} \vee z\right)$, then we can conclude that $x=y_{1} \wedge y_{2}$ and $z=y_{1} \wedge y_{2}$. Hence, $x$ and $z$ are indeed equivalent and we can replace $z$ with $x$ and vice-versa. Observe that, by suitable resolution operations, we could easily derive the clauses $(x \vee \neg z) \wedge(\neg x \vee z)$, obtaining the same conclusion.

The previous example suggests a pattern-matching approach for identifying a set $E$ of variables that can be expressed as a Boolean function $f$ of some other set $S$ of variables (i.e., the support set) and thus replace the variables in set $E$ by a single variable. This approach is simply too time consuming for sets $S$ of arbitrary size, and so we restrict $S$ to be of size 2, i.e. we only consider Boolean functions of two variables.

If the support set $S$ is restricted to be of size 2, it becomes feasible to enumerate all possible Boolean functions of 2 variables, and determine the irredundant CNF formulas associated with each Boolean function. This information is shown in Table 1. (Observe that the missing six boolean functions are either constant 0 or 1, the actual function of a single variable or of its complement.) We can now apply a straightforward pattern matching algorithm to a CNF formula, and identify sets $E$ of variables with a common two-variable support set. These variables can then be replaced by a single variable. Clearly, after variable replacement we can apply well-known formula simplification techniques [3].

Other researchers [1] have observed the existence of variable equivalences based on support sets, but on arbitrary instances of SAT (i.e. not in CNF format). Moreover, the underlying approach of $[1,4]$ is significantly more time consuming (indeed exponential in the worst-case) than the one proposed above. 
Table 1. Two-variable CNF formulas

\begin{tabular}{|l|l|}
\hline Boolean function & CNF formula \\
\hline$x \equiv f_{1}(a, b)=a \wedge b$ & $(a \vee \neg x) \wedge(b \vee \neg x) \wedge(\neg a \vee \neg b \vee x)$ \\
\hline$x \equiv f_{2}(a, b)=a \vee b$ & $(\neg a \vee x) \wedge(\neg b \vee x) \wedge(a \vee b \vee \neg x)$ \\
\hline$x \equiv f_{3}(a, b)=a \leftrightarrow b$ & $(a \vee b \vee x) \wedge(a \vee \neg b \vee \neg x) \wedge(\neg a \vee \neg b \vee x) \wedge(\neg a \vee b \vee \neg x)$ \\
\hline$x \equiv f_{4}(a, b)=\neg a \wedge b$ & $(\neg a \vee \neg x) \wedge(b \vee \neg x) \wedge(a \vee \neg b \vee x)$ \\
\hline$x \equiv f_{5}(a, b)=a \wedge \neg b$ & $(a \vee \neg x) \wedge(\neg b \vee \neg x) \wedge(\neg a \vee b \vee x)$ \\
\hline$x \equiv f_{6}(a, b)=\neg(a \wedge b)$ & $(a \vee x) \wedge(b \vee x) \wedge(\neg a \vee \neg b \vee \neg x)$ \\
\hline$x \equiv f_{7}(a, b)=\neg(a \vee b)$ & $(\neg a \vee \neg x) \wedge(\neg b \vee \neg x) \wedge(a \vee b \vee x)$ \\
\hline$x \equiv f_{8}(a, b)=\neg(a \leftrightarrow b)$ & $(a \vee b \vee \neg x) \wedge(a \vee \neg b \vee x) \wedge(\neg a \vee b \vee x) \wedge(\neg a \vee \neg b \vee \neg x)$ \\
\hline$x \equiv f_{9}(a, b)=a \vee \neg b$ & $(\neg a \vee x) \wedge(b \vee x) \wedge(a \vee \neg b \vee \neg x)$ \\
\hline$x \equiv f_{10}(a, b)=\neg a \vee b$ & $(a \vee x) \wedge(\neg b \vee x) \wedge(\neg a \vee b \vee \neg x)$ \\
\hline
\end{tabular}

Another interesting result, is that the equivalence reasoning conditions proposed by C.-M. Li in [2] are also superseded by support set equivalences and selective resolution (see $[1,3]$ for a definition). We should observe, in particular, that the utilization of function $f_{3}$ on two variables $x=(a \leftrightarrow b)$ and $y=(a \leftrightarrow b)$, for deriving the equivalence between $x$ and $y$, corresponds to Li's inference rule (5) [2], the other rules being superseded by selective resolution, unit-clause rule and two-variable equivalence.

Despite the potential interest of identifying support sets of variables in a CNF formula, in the next section a further generalization is proposed, that subsumes variable equivalences based on two-variable support sets.

\subsection{Generalized Inference of Binary Clauses}

In this section we propose to study subsets of clauses for inferring binary clauses, which not only identify support set equivalences, but also provide more general conditions for deriving binary clauses. In what follows, all proposed conditions can be explained by resorting to resolution. However, it is in general extremely hard to decide to which clauses the resolution operation should be applied to, being computationally infeasible to apply the resolution operation to all possible pairs of clauses. The objective of studying sets of clauses is to indirectly select to which sets of clauses the resolution operation should be applied to.

Moreover, the reasoning technique to be described below is categorized in terms of how many binary clauses and ternary clauses are involved. Clearly, the size and number of $k$-ary clauses involved could be made arbitrary, but the computational overhead could become prohibitive. In general each proposed condition is classified as being of the form $\mathrm{mB} / \mathrm{nT}$, meaning that $m$ binary clauses and $n$ ternary clauses are involved.

Let us start by considering an illustrative example. Let $\varphi_{c}=\left(y_{1} \vee \neg x\right) \wedge$ $\left(y_{2} \vee \neg x\right) \wedge\left(\neg y_{1} \vee \neg y_{2} \vee z\right)$ be a sub-formula. The application of the resolution operation between the three clauses allows deriving $(\neg x \vee z)$. Similarly, for the sub-formula $\varphi_{d}=\left(y_{1} \vee \neg z\right) \wedge\left(y_{2} \vee \neg z\right) \wedge\left(\neg y_{1} \vee \neg y_{2} \vee x\right)$, the resolution operation 
Table 2. Rules for Inferring Binary/Unit Clauses

\begin{tabular}{|l|r|}
\hline Clause Pattern & Inferred Clause $(\mathrm{s})$ \\
\hline \hline$\left(l_{1} \vee \neg l_{2}\right) \wedge\left(l_{1} \vee \neg l_{3}\right) \wedge\left(l_{2} \vee l_{3} \vee l_{4}\right)$ & $\left(l_{1} \vee l_{4}\right)$ \\
\hline$\left(l_{4} \vee \neg l_{2}\right) \wedge\left(l_{1} \vee \neg l_{3}\right) \wedge\left(l_{2} \vee l_{3} \vee l_{4}\right)$ & $\left(\neg l_{3} \vee l_{4}\right),\left(l_{1} \vee l_{4}\right)$ \\
\hline$\left(l_{1} \vee l_{2} \vee l_{3}\right) \wedge\left(\neg l_{1} \vee \neg l_{2} \vee l_{3}\right) \wedge\left(l_{1} \vee \neg l_{2} \vee l_{4}\right) \wedge\left(\neg l_{1} \vee l_{2} \vee l_{4}\right)$ & $\left(l_{3} \vee l_{4}\right)$ \\
\hline$\left(l_{1} \vee l_{2} \vee \neg l_{3}\right) \wedge\left(l_{1} \vee l_{2} \vee \neg l_{4}\right) \wedge\left(l_{1} \vee l_{2} \vee \neg l_{5}\right) \wedge\left(l_{3} \vee l_{4} \vee l_{5}\right)$ & $\left(l_{1} \vee l_{2}\right)$ \\
\hline$\left(l_{1} \vee l_{2}\right) \wedge\left(\neg l_{1} \vee l_{2} \vee l_{3}\right)$ & $\left(l_{2} \vee l_{3}\right)$ \\
\hline$\left(l_{1} \vee \neg l_{2}\right) \wedge\left(l_{1} \vee \neg l_{3}\right) \wedge\left(l_{1} \vee \neg l_{4}\right) \wedge\left(l_{2} \vee l_{3} \vee l_{4}\right)$ & $\left(l_{1}\right)$ \\
\hline$\left(l_{1} \vee l_{2} \vee \neg l_{3}\right) \wedge\left(l_{1} \vee l_{2} \vee \neg l_{4}\right) \wedge\left(l_{3} \vee l_{4}\right)$ & $\left(l_{1} \vee l_{2}\right)$ \\
\hline$\left(l_{1} \vee l_{2}\right) \wedge\left(\neg l_{1} \vee l_{3} \vee l_{4}\right) \wedge\left(l_{2} \vee l_{3} \vee \neg l_{4}\right)$ & $\left(l_{2} \vee l_{3}\right)$ \\
\hline
\end{tabular}

allows deriving the clause $(x \vee \neg z)$. It is interesting to observe that we have just illustrated a different approach for proving $x$ equivalent to $z$ for the first example of the previous section.

In general, let us consider a sub-formula of the form $\varphi_{s}=\left(l_{1} \vee \neg l_{2}\right) \wedge\left(l_{1} \vee\right.$ $\left.\neg l_{3}\right) \wedge\left(l_{2} \vee l_{3} \vee l_{4}\right)$, where $l_{1}, \ldots, l_{4}$ are any literals. The application of resolution allows deriving the binary clause $\left(l_{1} \vee l_{4}\right)$. Since 2 binary clauses and 1 ternary clause are involved in deriving the resulting binary clause, we say that $2 \mathrm{~B} / 1 \mathrm{~T}$ reasoning was applied. The other form of $2 \mathrm{~B} / 1 \mathrm{~T}$ reasoning is the following. Let the sub-formula be $\varphi_{t}=\left(l_{4} \vee \neg l_{2}\right) \wedge\left(l_{1} \vee \neg l_{3}\right) \wedge\left(l_{2} \vee l_{3} \vee l_{4}\right)$. Then, application of resolution allows deriving the binary clauses $\left(l_{3} \vee l_{4}\right)$ and $\left(l_{1} \vee l_{4}\right)$.

Additional forms of $\mathrm{mB} / \mathrm{nT}$ reasoning can be established. Due to efficiency concerns, our analysis will be restricted to $2 \mathrm{~B} / 1 \mathrm{~T}, 1 \mathrm{~B} / 1 \mathrm{~T}, 1 \mathrm{~B} / 2 \mathrm{~T}, 3 \mathrm{~B} / 1 \mathrm{~T}$ and $0 \mathrm{~B} / 4 \mathrm{~T}$. The resulting set of unit/binary clause inference rules is summarized in Table 2. Moreover, and from the previous examples and claims, we can readily conclude that support set variable equivalence (described in the previous section) is superseded by $2 \mathrm{~B} / 1 \mathrm{~T}$ and $0 \mathrm{~B} / 4 \mathrm{~T}$ reasoning.

\section{Experimental Results}

This section evaluates the practical application of the algebraic simplification techniques described in this paper. These techniques can be evaluated according to two main metrics:

- The ability to effectively simplify the original formula.

- The effective reduction in the amount of search, when using formula simplification techniques within a preprocessing engine for backtrack search SAT algorithms.

In this paper we concentrate on the ability to simplify the original formula. The reduction in the amount of search is analyzed in [3]. Moreover, the problem instances used in this section are also described in [3]

Table 3 shows the results, namely the number of variables and clauses before and after applying the simplification techniques. As can be concluded, for most of 
Table 3. Formula Simplification

\begin{tabular}{|l|r|r|r|r|}
\hline Instance & \multicolumn{2}{|c|}{ Initial Formula } & \multicolumn{2}{|c|}{ Final Formula } \\
\hline & Variables & Clauses & Variables & Clauses \\
\hline \hline bf1355-075 & 2180 & 6778 & 722 & 3075 \\
\hline bf2670-001 & 1393 & 3434 & 411 & 1252 \\
\hline ssa2670-130 & 1359 & 3321 & 440 & 1295 \\
\hline ssa2670-141 & 986 & 2315 & 283 & 883 \\
\hline \hline barrel6 & 2306 & 8931 & 519 & 2209 \\
\hline barrel7 & 3523 & 13765 & 805 & 3467 \\
\hline longmult5 & 2397 & 7431 & 1483 & 5180 \\
\hline longmult6 & 2848 & 8853 & 1824 & 6376 \\
\hline queueinvar12 & 1112 & 7335 & 1049 & 9884 \\
\hline queueinvar16 & 1168 & 6496 & 1088 & 7114 \\
\hline \hline c1908 & 1917 & 5096 & 658 & 2248 \\
\hline c1908_bug & 1919 & 5100 & 659 & 2250 \\
\hline c2670 & 2703 & 6756 & 1220 & 3725 \\
\hline c2670_bug & 2708 & 6696 & 1162 & 3553 \\
\hline \hline dlx2_cc_bug02 & 1515 & 12808 & 1486 & 13965 \\
\hline dlx2_cc_bug08 & 1515 & 12808 & 1486 & 13890 \\
\hline
\end{tabular}

the instances considered, large reductions in the number of variables and clauses can be achieved. In some cases (e.g. barrel6 and barrel7) the final number of variables is one fourth of the original number. Similar reductions can be observed in the number of clauses. Nevertheless, for some instances (e.g. queueinvar16 and dlx2_cc_bug08) the amount of simplification in the number of variables is negligible. In these cases, it is interesting to observe an increase in the number of clauses, due to the application of clause inference techniques (see Section 3.2).

\section{Conclusions}

This paper proposes new techniques for the algebraic simplification of propositional formulas. These techniques have been incorporated into a preprocessing system to be used with any SAT algorithm. Preliminary experimental results, obtained on real-world instances of SAT, clearly demonstrate the effectiveness of the proposed techniques, allowing significant reductions in the sizes of the resulting CNF formulas.

Despite the promising results obtained, and given the amount of simplification achieved on most problem instances, the next natural step is to incorporate the same algebraic simplification techniques into backtrack search SAT algorithms, to be applied during the search. 


\section{References}

1. J. F. Groote and J. P. Warners. The propositional formula checker heerhugo. Technical Report SEN-R9905, CWI, January 1999. 538, 539

2. C.-M. Li. Integrating equivalency reasoning into davis-putnam procedure. In Proceedings of the National Conference on Artificial Intelligence, August 2000. Accepted for publication. 537, 539

3. J. P. Marques-Silva. Algebraic simplification techniques for propositional satisfiability. Technical Report RT/01/2000, INESC, March 2000. 537, 538, 539, 540

4. G. Stålmarck. A system for determining propositional logic theorems by applying values and rules to triplets that are generated from a formula, 1989. Swedish Patent 467076 (Approved 1992), US Patent 5276897 (approved 1994), European Patent 0403454 (approved 1995). 538 